\title{
PEPPER (C. ANNUUM LINN) EXTRACT AMELIORATES ALTERATIONS TO NEURONAL CHOLINERGIC AND PURINERGIC ENZYME ACTIVITIES IN ROTENONE-INTOXICATED RAT MODEL OF PARKINSON'S DISEASE
}

\author{
Ogunruku O. O.* and Ogunyemi B. O. \\ Department of Biochemistry and Molecular Biology, Obafemi Awolowo University, Ile-Ife, Nigeria. \\ *Author for correspondence. E-mail: omodesola.oluwafisayo@gmail.com, Tel.: +234 8092083337 \\ (Received: $4^{\text {th }}$ March, 2019; Accepted: $23^{\text {rd }}$ March, 2019)
}

\section{ABSTRACT}

\begin{abstract}
The study evaluated the cognitive enhancing properties of the ethylacetate fraction of pepper, Capsicum annuum, in rotenone-intoxicated rat model of Parkinson's disease. Rats were divided into seven groups comprising normal rats, rat model of Parkinson's disease (PD) induced with intraperitoneal injection of rotenone $(2 \mathrm{mg} / \mathrm{kg})$, rotenone-intoxicated rats treated with $50,100,200 \mathrm{mg} / \mathrm{kg} /$ day of ethyl acetate fraction of C. annuum; rotenone-intoxicated rats treated with levodopa, and rotenone-intoxicated rats treated with levodopa plus $100 \mathrm{mg} / \mathrm{kg}$ C. annuum extract for 25 days. Subsequently, the spatial memory index of the rats was assessed in a Y-maze test. Thereafter, the activities of acetylcholinesterase, adenosine triphosphate diphosphohydrolase (ATPdase) and ecto-5'-nucleotidase were assayed in the rat brain homogenate. Results showed that there was a significant reduction in memory index and elevation in the activities of acetylcholinesterase, ATPDase and ecto-5'-nucleotidase in rotenone-intoxicated rats, which were significantly ameliorated in rotenone-intoxicated rats co-treated with ethyl acetate fraction of C. annuum in a dosedependent manner. Hence, it can be inferred from this study that ethyl acetate fraction of $C$. annuum exhibited protective properties against rotenone-induced model of Parkinson's disease in rats and could be a source of neuroprotective compounds.
\end{abstract}

Key words: Rotenone, Capsicum annuum, Acetylcholinesterase, Cognitive enhancement.

\section{INTRODUCTION}

Parkinson's disease (PD) is an age related, neurodegenerative disorder affecting $1 \%$ of the population above 60 years (McGregor and Nelson, 2019). The advancing age of the populace is a major risk factor as it is expected that the number of people seeking treatment for PD will dramatically increase over the next several decades. The socioeconomic impact associated with the global burden of this disease has brought a dire need to find both preventive and palliative measure of treatment for sufferers.

Chronic exposure to environmental neurotoxins particularly pesticides such as rotenone is associated with an increased risk of sporadic Parkinson's disease (PD) incidence (Nandipati and Livtan, 2016). The rotenone model of PD reproduces many features of the disease (Javed et al., 2016) all of which are a highly reproducible lesion of the nigrostriatal dopamine system associated with PD pathology, allowing for the assessment of pathogenic pathways and experimental therapeutic interventions.
Parkinson's disease is characterized by the loss of dopaminergic neurons in the substantia nigra pars compacta region of the brain leading to depletion in the dopamine levels. Depletion of dopamine, a neurotransmitter involved in coordinated movement, results in impaired motor performance (Hauser and Hastings, 2013). PD is traditionally seen as a motor syndrome secondary to nigrostriatal dopaminergic denervation, earlier reviews emphasize on non-motor features such as cognitive impairment which is better explained by multi-system neurodegeneration that extends beyond the loss of dopaminergic nigral neurons (Bohnen and Albin, 2010).

The neurotransmitter acetylcholine, plays a major role in cognition and memory (Xu et al., 2008) and a significant loss of nucleus basalis of Meynet (nbM) cholinergic neurons has been reported in PD (Bohnen and Albin, 2009). Elevated activity of acetylcholinesterase, the enzyme that hydrolyses acetylcholine at the synapse of cholinergic neurons, is a major culprit in dementia process (Contestabile, 2011). Treatments with cholinesterase inhibitors have been found to have 
a positive correlation with improved cognitive abilities with modest and significant therapeutic effects (Herrmann et al., 2011).

Adenosine triphosphate and its breakdown product adenosine are extra communication channels in the purinergic signaling pathway, alterations in this pathway have been implicated in the etiology of cognitive dysfunction as well as the movement disorders associated with Parkinson's disease. Inhibition of the enzymes involved in extracellular ATP and adenosine breakdown could improve the cognitive decline and motor symptoms of this disease (Burnstock, 2013).

Pepper or Chili a genus of related plants belongs to the solanaceae family of which the specie Capsicum annuum is included. Capsicum annuum L. is an herbaceous annual plant with glaborous pubescent, lanceolate leaves, white flowers and fruits (Evans, 1997). The fruits with seeds are used commonly as spices due to their pungent flavor. Oboh and Ogunruku (2010) reported the ability of pepper diet to protect brain cells from oxidative damage. It was also reported by Nielsen et al., (2013) that eating pepper is associated with $19 \%$ lower risk of PD in 500 diagnosed patients. Consequently, C. annuum could be exploited as a readily available and cheap dietary means of managing PD and its symptoms. Therefore, this study sought to investigate the cognitive enhancing properties of the Capsicum annuum Linn in a parkinsonian rat model induced by rotenone.

\section{MATERIALS AND METHODS \\ Materials}

Sample Collection: Fresh pepper samples (C. annuum Linn.) were collected from Gbogan area of Osun State, Nigeria. Identification and authentication of the samples were carried out at the Department of Botany, Obafemi Awolowo University, Ile-Ife, Nigeria with voucher number 17533 given to the plant. The samples were freezedried and blended into powdery form.

Chemicals and Reagents: Chemicals and reagents used such as adenosine-5'monophosphate, adenosine-5'-triphosphate, dinitrophenyl hydrazine (DNPH), 5,5'-dithio-bis (2-nitrobenzoic) acid (DNTB), hydrogen peroxide, sodium dodecyl sulphate, potassium chloride, calcium chloride, sodium carbonate, acetylthiocholine iodide, were sourced from Sigma Aldrich, Inc., (St Louis, MO). Rotenone was obtained from Chem Cruz, (Santa Cruz Biotechnology Inc., Dallas). Unless stated otherwise, all other chemicals and reagents were of analytical grades and water was glass distilled.

Experimental Animals: Adult Wistar rats (150 $\mathrm{g}-250 \mathrm{~g}$ ) were obtained from the Animal House, College of Health Sciences, Obafemi Awolowo University, Ile Ife, Osun State, Nigeria. The animals were kept under ambient conditions in metal cages. They were acclimatized for two weeks before the commencement of the experiment, fed with standard commercial pellet and allowed access to water ad libitum. The rats were housed under standard conditions, at natural light and dark cycle.

\section{Methods \\ Extract Preparation}

A solvent mixture of methanol and water (1:1, $\mathrm{v} / \mathrm{v})$ containing $0.5 \mathrm{M} \mathrm{HCl}$ was used to extract $2 \mathrm{~kg}$ of the pepper fruits $(1: 4 \mathrm{w} / \mathrm{v})$ for 48 hours and then filtered using filter paper (Whatman no. 2), the filtrate was concentrated under vacuum at 40 ${ }^{\circ} \mathrm{C}$. The crude extract was then re-dissolved in distilled water and partitioned with equal volume of ethylacetate. The ethylacetate fraction was concentrated under vacuum at $40{ }^{\circ} \mathrm{C}$ in a rotary evaporator until about $95 \%$ of the solvent had been evaporated and stored at $4{ }^{\circ} \mathrm{C}$ until use. The percentage yield was calculated to be $0.16 \%$.

\section{Experimental Design}

The rats were randomly assigned into seven groups $(n=6)$. Parkinsonism was induced in rats by intraperitoneal administration of $2 \mathrm{mg} / \mathrm{kg}$ body weight (BWT) of rotenone prepared in a mixture of $1 \%$ dimethyl sulfoxide (DMSO) in sunflower oil. The experimental rats were treated orally as follows: Group 1-Basal control; Group 2-Disease control (2 mg/kg BWT rotenone only); Groups 3, 4 and 5 were treated with rotenone and 50, 100, $200 \mathrm{mg} / \mathrm{kg}$ BW'T of the extract respectively; Group 6 was treated with rotenone and $30 \mathrm{mg} / \mathrm{kg}$ BWT levodopa as the control drug; Group 7 was 
treated with rotenone, the extract $(100 \mathrm{mg} / \mathrm{kg}$ BW'T) and levodopa (30 mg/kg BWT). The extract was daily administered orally, 3 hours prior to rotenone administration; each animal received 5 doses of rotenone per week. The weights of the rats were monitored at an interval of three days throughout the duration of the study ( 25 days). At the end of the experiment, animals were sacrificed by cervical dislocation and the brain tissue was quickly excised, blotted with filter paper, weighed and rinsed in cold saline solution. The tissues were homogenized in phosphate buffered saline and thereafter preserved at $-20^{\circ} \mathrm{C}$ until further analysis.

\section{Cognition Test (Y-maze)}

Cognitive function was assessed via the Y-maze test during the last week of the experiment. Tests were conducted using the method of Hsiao et al., (1995). Each arm of the Y-maze was $35 \mathrm{~cm}$ long, 5 $\mathrm{cm}$ wide, and $10 \mathrm{~cm}$ high, the Y-maze was rotated by $45^{\circ}$ and the distal cues differed between the forced and the spontaneous alternation test. This test consisted of a single 5 min trial, in which the mouse was allowed to explore all three arms of the Y-maze. The mouse that climbed on the maze walls was immediately returned to the abandoned arm. Spontaneous Alternation (\%) was defined as consecutive entries in 3 different arms (ABC), divided by the number of possible alternations (total arm entries minus 2).

\section{Acetylcholinesterase Activity Assay}

The acetylcholinesterase activity was determined according to the method of Ellman et al. (1961). Briefly, the reaction mixture containing $200 \mu \mathrm{l}$ of the rat's brain crude homogenate supernatant $(0.342-0.435 \mathrm{mg}$ of protein) in $500 \mu \mathrm{l}$ of $0.1 \mathrm{M}$ phosphate buffer ( $\mathrm{pH} 8.0), 100 \mu$ l solution of 3.3 mM 5,5'-dithio-bis(2-nitrobenzoic) acid (DNTB) and $500 \mu \mathrm{l}$ of phosphate buffer, $(\mathrm{pH}$ 8.0) was incubated at $25{ }^{\circ} \mathrm{C}$ for $20 \mathrm{~min}$. Acetylthiocholine iodide (100 $\mu$ l of $0.05 \mathrm{mM}$ solution) was added as the substrate. Change in absorbance at $412 \mathrm{~nm}$ was read every $30 \mathrm{sec}$ for a period of 3 mins.

\section{ATPDase Activity Assay}

The ATPDase enzymatic activity was determined in whole brain crude homogenate supernatant using the method of Schetinger et al., (2000) with slight modification. The assay mixture contained 5
$\mathrm{mM} \mathrm{CaCl}_{2}, 5 \mathrm{mM} \mathrm{KCl}, 0.1 \mathrm{mM}$ EDTA, $50 \mathrm{mM}$ Tris-HCl buffer ( $\mathrm{pH}$ 7.4), in a final volume of 200 $\mu \mathrm{l} .20 \mu \mathrm{l}$ of brain crude homogenate supernatant $(0.028-0.036 \mu \mathrm{g}$ of protein) was added, and preincubated at $37^{\circ} \mathrm{C}$ for $10 \mathrm{~min}$. The reaction was initiated by the addition of ATP to obtain a final concentration of $1.0 \mathrm{mM}$, and then incubated for $20 \mathrm{~min}$. The reaction was stopped by the addition of $200 \mu \mathrm{l}$ of $10 \%$ trichloroacetic acid (TCA) to obtain a final concentration of $5 \%$. After cooling on ice for $10 \mathrm{~min}, 40 \mu \mathrm{l}$ of the reaction mixture was assayed for the release of inorganic phosphate by the method of Fiske and Subbarow (1925) in which the phosphomolybdate complex formed is reduced by ascorbate to produce the characteristic blue-green colour that is detected at $650 \mathrm{~nm}$ and $\mathrm{NaH}_{2} \mathrm{PO}_{4}$ was used as the standard.

\section{Ecto-5'-nucleotidase Activity Assay}

Ecto-5'-nucleotidase activity was determined in whole brain crude homogenate supernatant as described by Heymann et al., (1984). The assay mixture consisted of $10 \mathrm{mM} \mathrm{MgSO}_{4}$ and $100 \mathrm{mM}$ Tris-HCl buffer ( $\mathrm{pH} 7.5$ ), $20 \mu \mathrm{l}$ of brain crude homogenate supernatant preparation (0.038 $0.048 \mu \mathrm{g}$ of protein) were added to the reaction mixture and pre-incubated at $37{ }^{\circ} \mathrm{C}$ for 10 mins. The reaction was initiated by the addition of AMP to obtain a final concentration of $2 \mathrm{mM}$ and incubated for 20 mins. The reaction was stopped by the addition of $200 \mu \mathrm{l}$ of $10 \%$ trichloroacetic acid to obtain a final concentration of $5 \%$. Thereafter, the tubes were chilled on ice for 10 mins. The released inorganic phosphate was assayed for by the method of Fiske and Subbarow (1925).

\section{Statistical Analysis}

Data obtained were subjected to statistical analysis using GraphPad ${ }^{\circledR}$ Prism Statistical Package Version 7 and expressed as mean \pm standard error of mean (SEM). Data obtained from cylinder test was subjected to two-way analysis of variance (ANOVA) followed by Tukey test while all other data were compared using one-way ANOVA followed by Tukey test. Significance was accepted at $P<0.05$.

\section{RESULTS}

The result for spontaneous alternation as a 
measure of memory index in the treated rats is presented in figure 1 . There was a significant $(\mathrm{P}<0.05)$ decrease in spontaneous alternation resulting from intraperitoneal rotenone administration $(2 \mathrm{mg} / \mathrm{kg}$ BWT) when compared with the basal control in all the experimental days. Treatment with ethylacetate fraction of $C$. annuum at $50 \mathrm{mg} / \mathrm{kg}$ BWT resulted in a progressive increase in spontaneous alternation score reaching significance $(\mathrm{P}<0.05)$ on the last day of experiment while significant $(\mathrm{P}<0.05)$ increase was observed for extract at 100 and 200 $\mathrm{mg} / \mathrm{kg}$ BWT across the experimental days. Furthermore, the control drug levodopa (30 $\mathrm{mg} / \mathrm{kg} \mathrm{BWT}$ ) significantly $(\mathrm{P}<0.05)$ increased the spontaneous alternation score across the days of experiment when compared to the group treated with rotenone only. Rats to which both levodopa and extract at $100 \mathrm{mg} / \mathrm{kg}$ BWT were administered also exhibited increased spontaneous alternation when compared to the rats to which only rotenone was administered.

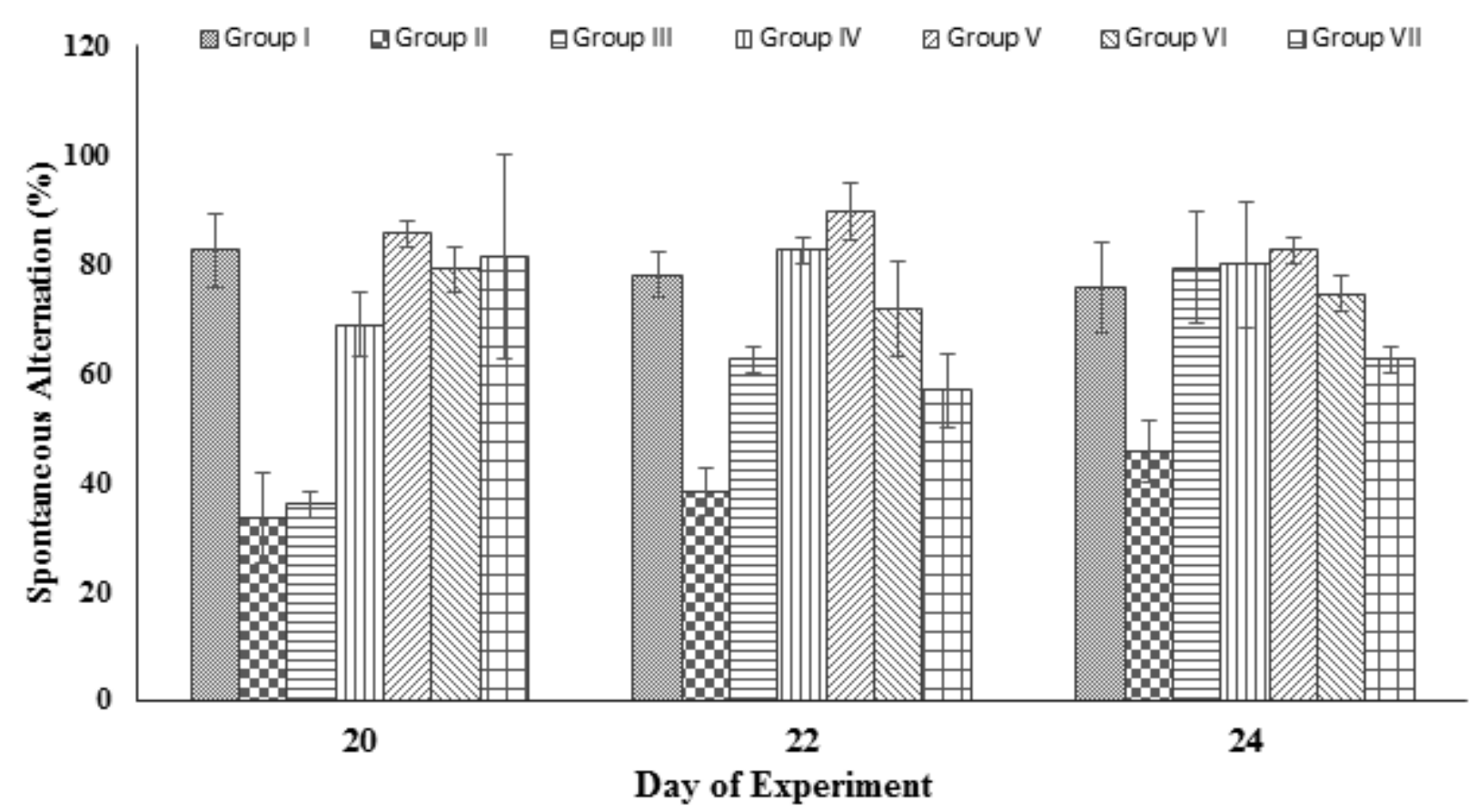

Figure 1: Cognitive Enhancement in Rotenone-intoxicated Rats by Capsicum annuum

Bars with same letters are not significantly $(\mathrm{P}<0.05)$ different.

Values are expressed as mean \pm SEM $(n=6)$. (a) and (b) represent significant difference at $\mathrm{P}<0.05$ when compared to the control and disease control groups respectively. Group 1: Basal control; Group 2: Disease control (2 $\mathrm{mg} / \mathrm{kg}$ BW'T Rotenone); Group 3: Rotenone (2 mg/kg BWT) + C. annuum (50 mg/kg BWT); Group 4: Rotenone (2 mg/kg BWT) + C. annuum (100 $\mathrm{mg} / \mathrm{kg}$ BWT); Group 5: Rotenone (2 $\mathrm{mg} / \mathrm{kg}$ BW'T) + C. annuum (200 mg/kg BWT); Group 6: Rotenone (2 mg/kg BWT) + Levodopa (30 $\mathrm{mg} / \mathrm{kg}$ BWT); Group 7: Rotenone (2 mg/kg BWT $)+$ Levodopa (30 mg/kg BW'T) + C. annuum (100 mg/kg BWT).
The brain cholinesterase activities in treated rats are presented in figure 2 . There was an increase in AChE activity in rotenone-administered rats compared to the control. However, treatment with ethylacetate fraction of C. annuum at 200 $\mathrm{mg} / \mathrm{kg}$ BWT resulted in a significant $(\mathrm{P}<0.05)$ decrease in $\mathrm{AChE}$ activity compared to the rats to which only rotenone was administered. Nevertheless, rats to which both levodopa and extract $(100 \mathrm{mg} / \mathrm{kg}$ BWT) were administered resulted in a decrease in $\mathrm{AChE}$ activity below that of levodopa-administered rats. 


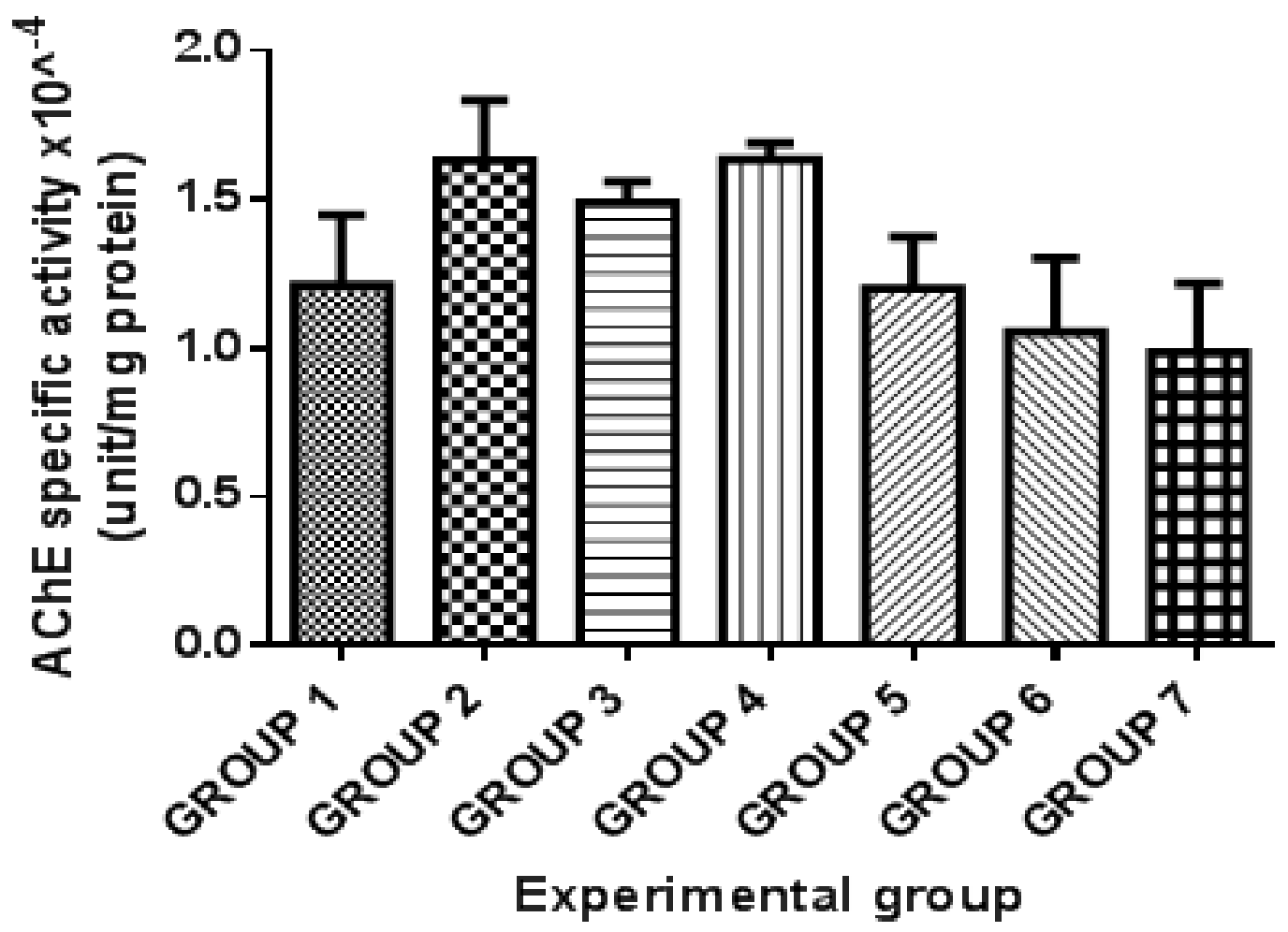

Figure 2: Effect of Capsicum annuum Diminishes on Brain Acetylcholinesterase in Rotenoneintoxicated Rats.

Values are expressed as mean $\pm \operatorname{SEM}(\mathrm{n}=6)$. (a) and (b) represent significant difference at $\mathrm{P}<0.05$ when compared to the control and disease control groups respectively. Group 1: Basal control; Group 2: Disease control (2 $\mathrm{mg} / \mathrm{kg}$ BWT Rotenone); Group 3: Rotenone ( $2 \mathrm{mg} / \mathrm{kg}$ BWT) + C. annuum (50 mg/kg BWT); Group 4: Rotenone (2 mg/kg BWT) + C. annuum (100 $\mathrm{mg} / \mathrm{kg}$ BWT); Group 5: Rotenone (2 mg/kg BWT $)+$ C. annuum (200 mg/kg BWT); Group 6: Rotenone (2 mg/kg BWT) + Levodopa (30 $\mathrm{mg} / \mathrm{kg}$ BWT); Group 7: Rotenone (2 mg/kg BWT $)+$ Levodopa $(30 \mathrm{mg} / \mathrm{kg} \mathrm{BWT})+C$. annuum (100 mg/kg BWT).

Figure 3 presents the impact of extracts on brain adenosine triphosphate diphosphorylase (ATPDase) activity in rotenone-induced Parkinsonism in rats. There was a significant $(\mathrm{P}<0.05)$ increase in ATPDase activity in rats to which rotenone had been intraperitoneally administered compared to control. However, treatment with ethylacetate fraction of $C$. annuum (50-200 mg/kg BWT) resulted in a dosedependent reduction in ATPDase activity compared with the rotenone-treated rats. Furthermore, rats that were administered the extract at $200 \mathrm{mg} / \mathrm{kg}$ BWT, as well as rats to which both levodopa and extract $(100 \mathrm{mg} / \mathrm{kg}$ BWT) were administered resulted in a significant $(\mathrm{P}<0.05)$ reduction in ATPDase activity compared with rats to which only rotenone was administered. 


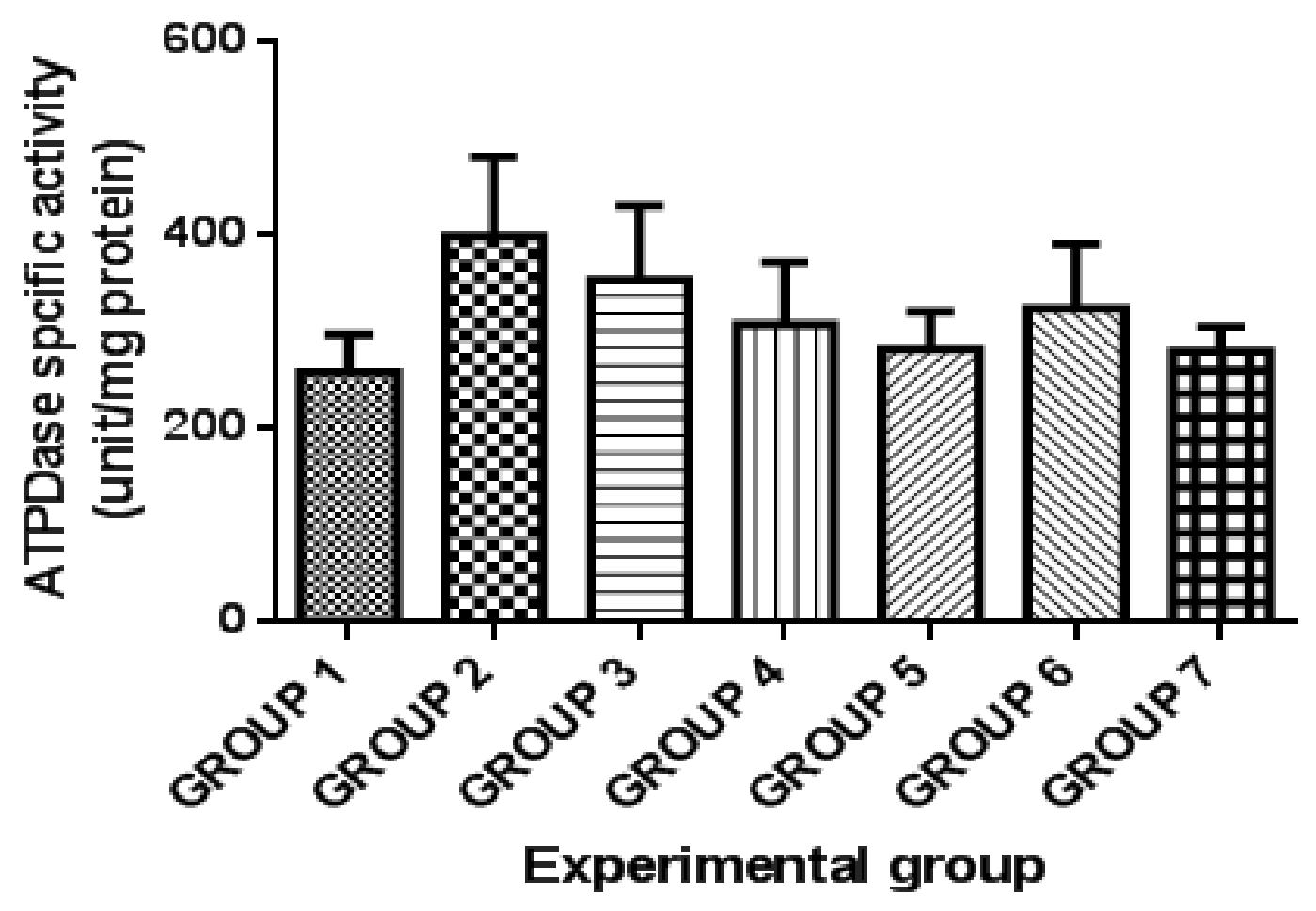

Figure 3: Effect of Capsicum annuum on the Brain ATPDase in Rotenone-intoxicated Rats

Values are expressed as mean \pm SEM $(n=6)$. (a) and (b) represent significant difference at $\mathrm{P}<0.05$ when compared to the control and disease control groups respectively. Group 1: Basal control; Group 2: Disease control (2 $\mathrm{mg} / \mathrm{kg}$ BW'T Rotenone); Group 3: Rotenone (2 mg/kg BWT) + C. annuum (50 mg/kg BWT); Group 4: Rotenone $(2 \mathrm{mg} / \mathrm{kg}$ BWT $)+$ C. annuum (100 $\mathrm{mg} / \mathrm{kg}$ BWT); Group 5: Rotenone (2 mg/kg BWT $)+$ C. annuum (200 mg/kg BW'T); Group 6: Rotenone $(2 \mathrm{mg} / \mathrm{kg}$ BWT) + Levodopa (30 $\mathrm{mg} / \mathrm{kg}$ BWT); Group 7: Rotenone $(2 \mathrm{mg} / \mathrm{kg}$ BWT $)+$ Levodopa $(30 \mathrm{mg} / \mathrm{kg}$ BWT $)+C$. annuum $(100 \mathrm{mg} / \mathrm{kg}$ BWT).
The ecto-5'-nucleotidase activity in the treated rats were assessed and presented in figure 4 . There was a significant $(\mathrm{P}<0.05)$ increase in ecto-5'nucleotidase activity in rotenone-administered rats compared to control rats. However, treatment with ethylacetate fraction of $C$. annuum $(50-200 \mathrm{mg} / \mathrm{kg}$ BWT) resulted in dosedependent reduction in ecto-5'-nucleotidase activity reaching a significant level $(\mathrm{P}<0.05)$ at 200 $\mathrm{mg} / \mathrm{kg} \mathrm{BWT}$ when compared with the rotenonetreated rats. Nevertheless, rats to which both levodopa and $100 \mathrm{mg} / \mathrm{kg}$ BW'T of the extract were administered resulted into further decrease in ecto-5'-nucleotidase activity when compared with the rats to which only levodopa was administered. 


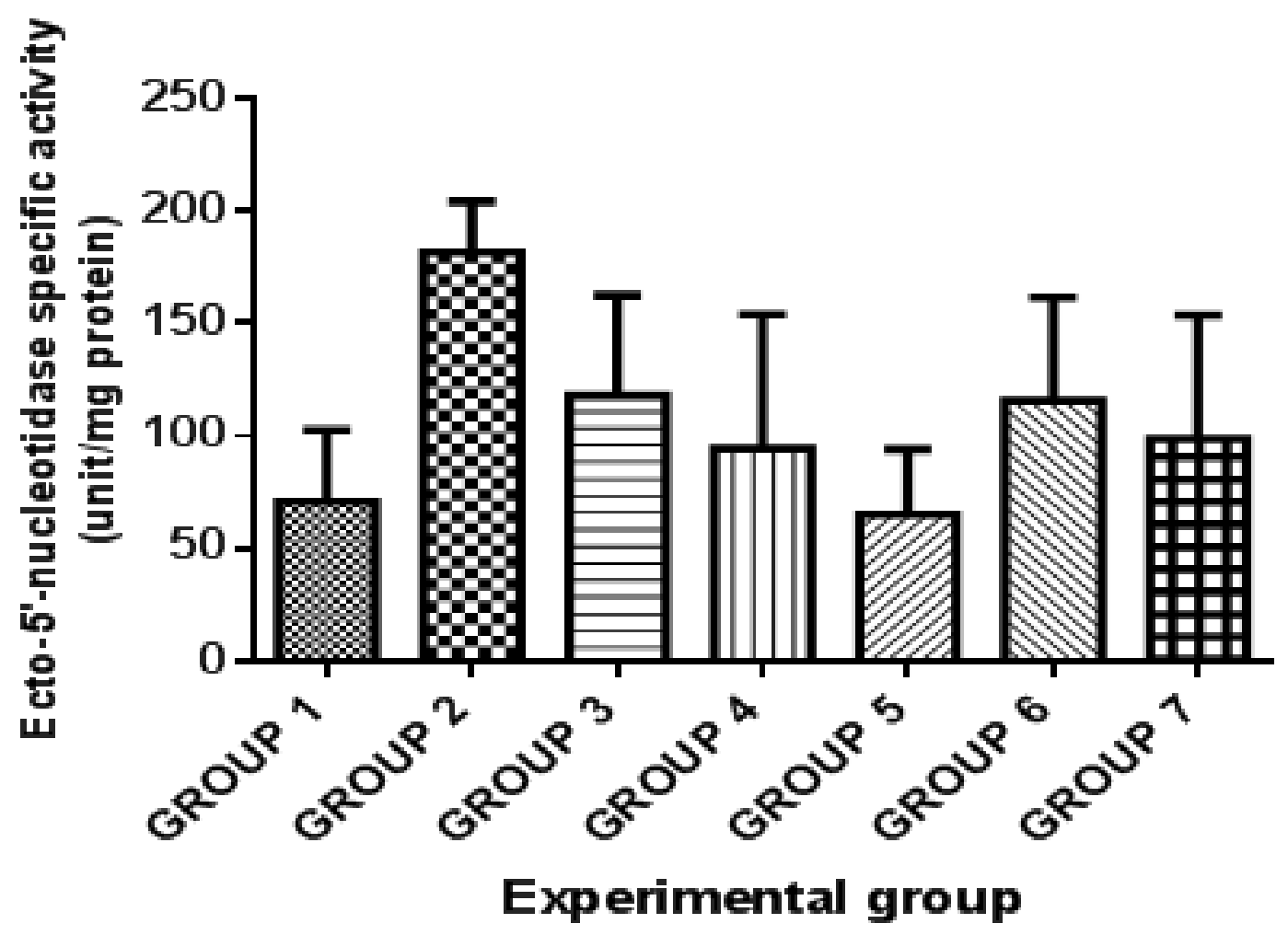

Figure 4: Effect of Capsicum annuum on Brain Ecto-5'-nucleotidase in Rotenone-intoxicated Rats

Values are expressed as mean \pm SEM ( $n=6)$. (a) and (b) represent significant difference at $\mathrm{P}<0.05$ when compared to the control and disease control groups respectively. Group 1: Basal control; Group 2: Disease control (2 $\mathrm{mg} / \mathrm{kg}$ BWT Rotenone); Group 3: Rotenone (2 mg/kg BWT) + C. annuum (50 mg/kg BWT); Group 4: Rotenone (2 mg/kg BWT) + C. annuum (100 $\mathrm{mg} / \mathrm{kg}$ BWT); Group 5: Rotenone (2 mg/kg BWT) + C. annuum (200 mg/kg BWT); Group 6: Rotenone (2 mg/kg BWT) + Levodopa (30 $\mathrm{mg} / \mathrm{kg}$ BWT); Group 7: Rotenone (2 mg/kg BWT $)+$ Levodopa (30 mg/kg BWT) + C. annuum (100 mg/kg BWT).

\section{DISCUSSION}

Cognitive decline is observed in early stages of Parkinsonism, it is well recognized that the cholinergic neurons are involved integrally in neurological functions such as learning, memory, sleep, and cognition, with acetylcholine playing a vast role in modulating these functions (Shinomol, 2011). Chronic administration of rotenone has been reported to cause altered cognition (Kaur et al., 2011). In this study, intraperitoneal rotenone administration brought about cognitive impairment as justified by decrease in the rats' spontaneous alternation as a measure of their memory index. The striatum contains a population of cholinergic interneurons, and there are indications that cholinergic neurotransmission plays a role in striatal function (Lecourtier and Kelly, 2007). This could have contributed to the cognitive decline so observed.

Dietary phytochemicals and supplementations using food extracts has been reported to influence cognition and learning in humans and animals either by protecting vulnerable neurons, enhancing neuronal function and stimulating neurogenesis (Kumar and Khanum, 2012). Treatment with ethylacetate fraction of $C$. annuum resulted in a significant $(\mathrm{P}<0.05)$ improvement in the memory index of rotenoneinduced rats. This observed cognitive enhancement by the extract of C. annum may be due to the extract's potentials in protecting against striatal cholinergic degeneration. Interestingly, Capsicum spp are constituents of decoctions 
prescribed traditionally as cognitive enhancers in southwestern Nigeria (Elufioye et al., 2012). Also Yang et al., (2015) reported that red pepper with moderate and severe pungency prevent memory deficit in rats. Furthermore, the levodopa (30 $\mathrm{mg} / \mathrm{kg} \mathrm{BWT}$ ) treated rats showed no decrease in their memory index. Levodopa has been reported to enhance working memory while impairing other complex functions (Merims and Giladi, 2008).

The neurotransmitter-acetylcholine plays a major role in cognition and memory and a strong correlation has been established between loss of acetylcholine at the synaptic cleft (i.e. a cholinergic deficit) and dementia ( $\mathrm{Xu}$ et al., 2008). Disturbances in cholinergic function have been suggested to mediate many of the symptoms associated with dementia (Bohnen and Albin, 2010). Rotenone has been shown to interfere with cholinergic transmission (Yasser et al., 2016). A significant $(\mathrm{P}<0.05)$ increase in $\mathrm{AChE}$ activity following rotenone administration was observed in this study (Figure 2). Elevated activity of acetylcholinesterase is a major culprit in dementia process (Contestabile, 2011). Inhibition of cholinesterases has been accepted as one of the therapeutic strategies for ameliorating cholinergic deficit, thus the use of acetylcholinesterase inhibitors has been shown to slow down the progression of dementia in Alzheimer's disease (AD) and Parkinson's disease (PD) patients. It is noteworthy that the result of this study indicates that ethylacetate extract of $C$. annuum suppresses significantly $(\mathrm{P}<0.05)$ the activities of $\mathrm{AChE}$. The observed inhibitory activity of the extract could be attributed to the constituent fatty acid esters as some are structurally similar to the enzyme substrate, actetylcholine, which can of course undergo acylation (cabamylation) to form a carbomyl-enzyme intermediate, a state in which the enzyme has a low affinity for its substrate (a pseudo-irreversible inhibition). Furthermore, the phenol-based constituents of this extract could act like the well-known anticholinesterase inhibitors (e.g. Donepezil, Rivastigmine, Diazinon and Malathion) due to the presence of phenol rings and hydrophobic moieties in their structure thus making them potent AChE inhibitors. The ability of plant phenolic extracts to inhibit acetylcholinesterase activity has been well documented; recent study from Oboh et al., (2016) reported the AChE inhibitory effect of phenolic extracts from Heinsera crinite leaves with potential neuroprotective capabilities. Furthermore, pepper extracts reportedly inhibited the activity of AChE with increasing extract concentrations (Ogunruku et al., 2014). The reference drug was able to significantly $(\mathrm{P}<0.05)$ reduce AChE activity when administered alone as well as when administered in combination with extract at $100 \mathrm{mg} / \mathrm{kg} \mathrm{BWT}$. This is in line with the study of Messripour and Shahidi (1990) where they reported that administration of levodopa (10 $\mathrm{mg} / \mathrm{kg}$ BWT) resulted in decrease in acetylcholinesterase activity in rat brain.

Alterations in the purinergic signaling have been implicated in the etiology of cognitive dysfunction (Ferre et al., 1997). Inhibition of the enzymes involved in extracellular ATP and adenosine breakdown could improve the cognitive decline and motor symptoms of this disease (Burnstock, 2013). In this study, groups treated with rotenone only had a significant $(\mathrm{P}<0.05)$ high activity level of these enzymes (ATPDase and ecto-5'-nucleotidase). Rotenone being a mitochondrial complex I inhibitor prevents ATP synthesis, and aside the function of ATP as a classical neurotransmitter as well as being a cotransmitter, ATP enables neuronal cells to maintain a resting membrane potential; depletion of which results in loss of neurotransmission in both the dopaminergic and cholinergic neurons of the striatum. Furthermore, the inhibition of ATPDase can reduce ATP hydrolysis; this is of neurological importance because neurodegenerative diseases such as PD are characterized by impaired mitochondrial function and reduced ATP level which is closely associated with impaired cognitive functions (Gutierres et al., 2012).

Significant amounts of extracellular adenosine in the brain parenchyma are due to the breakdown of ATP released from nerve and astroglia cells, which express cell surface ectonucleotidases with their catalytic site facing the extracellular space (Zimmermann, 2000). Ecto-5'-nucleotidase is responsible for metabolizing AMP to adenosine (Zimmermann, 2000). Adenosine is a 
neuromodulator that can inhibit the release of neurotransmitters such as acetylcholine, dopamine, serotonin and glutamate. Thus, inhibiting the activities of these enzymes could be of therapeutic importance in management of the cognitive decline associated with PD. In this study, extract administration resulted in a moderate dose-dependent inhibition of the enzymes involved in purinergic signaling (ATPDase and ecto-5'-nucleotidase activities) reaching a significant level $(\mathrm{P}<0.05)$ at $200 \mathrm{mg} / \mathrm{kg}$ BWT extract concentration. Several other phytochemicals especially caffeic acid, a natural phenolic compound found in many plants, fruits and vegetables have been reported to inhibit the activities of these enzymes. Akomolafe et al., (2017) reported that caffeic acid and caffeine inhibited the activities of ATPDase and ecto-5'nucleotidase in both cerebral cortex and whole brain in which the cerebral cortex was removed. Furthermore, treatment with levodopa only does not significantly reduce the activities of these enzymes but its combination with extract at 100 $\mathrm{mg} / \mathrm{kg}$ BWT led to further decrease in activity which was significant $(\mathrm{P}<0.05)$ for ATPDase; thus, suggesting a synergistic interaction.

\section{CONCLUSION}

This study showed that pepper (C. annuum) could play an important role in cognitive enhancement and ameliorating some biochemical impairments associated with PD. The extract at $200 \mathrm{mg} / \mathrm{kg}$ BWT and the combination of extract and standard drug (levodopa) showed synergistic effects. Based on these findings, it could be possible to develop a multifaceted therapeutic strategy in PD therapeutic management.

\section{Conflict of Interests}

The authors declare that there is no conflict of interests regarding the publication of this paper.

\section{Acknowledgements}

The authors thank the Tertiary Education Trust Fund (TETFund), Federal Government of Nigeria for research grants (VC/Vol. IX/224/TETFUND / DESS / OAU / ILEIFE/VOV.V/2014-2015/5) used in the execution of this study.

\section{REFERENCES}

Akomolafe, S. F., Akinyemi, A. J., Ogunsuyi, O. B., Oyeleye, S. I., Oboh, G., Adeoyo, O. O. and Allismith, Y. R. (2017). Effect of caffeine, caffeic acid and their various combinations on enzymes of cholinergic, monoaminergic and purinergic systems critical to neurodegeneration in rat brain-In vitro. Neurotoxicology, 62: 613.

Bohnen, N. I. and Albin, R. L. (2009). Cholinergic denervation occurs early in Parkinson disease. Neurology, 73(4): 256 LP-257.

Bohnen, N. I. and Albin, R. L. (2010). The cholinergic system and Parkinson disease. Behavioural Brain Research, 221(2): 564573.

Burnstock, G. (2013). Purinergic signaling: Pathophysiology and therapeutic potential. Keio Journal of Medicine, 62(3): 63-73.

Contestabile, A (2011): The history of the cholinergic hypothesis. Behavioral Brain Research, 221(2):334-340

Ellman, G. L., Courtney, K. D., Andres, V. and Featherstone, R. M. (1961). A new and rapid colorimetric determination of acetylcholinesterase activity. Biochemical Pharmacology, 7: 88-95.

Elufioye, T. O., Oladele, A. T., Cyril-Olutayo, C. M., Agbedahunsi J. M. and Adesanya S. A. (2012). Ethnomedicinal study and screening of plants used for memory enhancement and anti-aging in Sagamu, Nigeria. European Journal of Medicinal Plants, 2: 262-275.

Evans, W. C. (1997). Trease and Evans Pharmacognosy, W. B. Saunders, Londen, $14^{\text {th }}$ Edn. ISBN: 0-7020-1899-6.

Ferre, S., Fredholm, B. B., Morelli, M., Popoli, P. and Fuxe, K. (1997). Adenosinedopamine receptor-receptor interactions as an integrative mechanism in the basal ganglia. Trends in Neuroscience, 20:482487

Fiske, C. H. and Subbarrow, Y. (1925). The colourimetric determination of phosphorous. Journal of Biological Chemistry, 66: 375-400. 
Gutierres, J. M., Carvalho, F. B., Schetinger, M. R., Rodrigues, M. V., Schmatz, R. and Pimentel, V. C. (2012). Protective effects of anthocyanins on the ectonucleotidase activity in the impairment of memory induced by scopolamine in adult rats. Life Sciences, 91 (23): 1221-1228.

Hauser, D. N. and Hastings, T. G. (2013). Mitochondrial oxidative stress in Parkinson's disease and monogenic Parkinsonism. Neurobiological Disorders, 51:35-42.

Herrmann, N., Chau, S.A., Kircanski, I. and Lanctot, K. L. (2011). Current and emerging drug treatment options for Alzheimer's disease. Drugs, 71:2031.

Heymann, D., Reddington, M. and Kreutzeberg, G. W. (1984). Subcellular localization of 5 '-nucleotidase in rat brain. Journal of Neurochemistry, 43: 971-978.

Hsiao, K. K., Borchelt, D. R., Olson, K., Johannsdottir, R., Kitt, C. and Yunis, W. (1995). Age-related CNS disorder and early death in transgenic $\mathrm{FVB} / \mathrm{N}$ mice overexpressing Alzheimer amyloid precursor proteins. Neuron, 15: 1203-1218.

Javed, H., Azimullah, S., Abul Khair, S. B., Ojha, S., and Haque, M. E. (2016). Neuroprotective effect of nerolidol against neuroinflammation and oxidative stress induced by rotenone. BMC Neuroscience, 17(1):1-12.

Kaur, H., Chauhan, S and Sandhir, R. (2011). Protective effect of lycopene on oxidative stress and cognitive decline in rotenone induced model of Parkinson's disease. Neurochemistry Research, 36: 1435-1443

Kumar, G. P. and Khanum, F. (2012). Neuroprotective potential of phytochemicals. Pharmacognosy reviezes, 6(12):81-90.

Lecourtier, L. and Kelly, P. H. (2007). A conductor hidden in the orchestra? Role of the habenular complex in monoamine transmission and cognition. Neuroscience and Biobehavioural Reviews, 31:658-72

McGregor, M. M. and Nelson, A. B. (2019).
Circuit mechanisms of Parkinson's disease. Neuron, 101(6):1042-1056.

Merims, D. and Giladi, N. (2008). "Dopamine dysregulation syndrome, addiction and behavioural changes in Parkinson's disease". Parkinsonism Related Disorder, 14 (4): 273-280.

Messripour, M. and Shahidi, Z. (1990). Short and long-term effects of L-dopa administration on striatal acetylcholinesterase activity. Molecular and Chemical Neuropathology, 13 (3): 217-224.

Nandipati, S. and Litvan, I. (2016). Environmental Exposures and Parkinson's Disease. International Journal of Environmental Research and Public Health, 13(9): 881.

Nielsen, S. S., Franklin, G. M., Longstreth, W. T., Swanson, P. D., and Checkoway, H. (2013). Nicotine from edible Solanaceae and risk of Parkinson disease (ANA-12-1625). Annals of Neurology, 74(3): 472-477.

Oboh, G. and Ogunruku, O. O. (2010). Cyclophosphamide-induced oxidative stress in brain: protective effect of hot short pepper (Capsicum frutescens $L$. Var. abbreviatum). Experimental and Toxicological Pathology, 62:227-233.

Oboh, G., Nwanna, E. E., Oyeleye, S. I., Olasehinde, T. A., Ogunsuyi, O. B. and Balogun, A. A. (2016). In vitro neuroprotective potentials of aqueous and methanolic extracts from Heinsia crinita leaves. Food Science and Human Wellness, 5: 95-102.

Ogunruku, O. O., Oboh, G. and Ademosun, A. O. (2 $\left.\begin{array}{llll} & 0 & 1 & 4\end{array}\right)$. Water extractable phytochemicals from peppers (Capsicum spp.) inhibit acetylcholinesterase and butyrylcholinesterase activities and prooxidants induced lipid peroxidation in ratbrain in vitro. International Journal of Food Science, bttp://dx.doi.org/10.1155/2014/60561 8.

Schetinger, M. R. C., Porto, N., Moretto, M. B., Morsch, V. M., Vieira, V., Moro, F., Neis, R. T., Bittencourt, S., Bonacorso, H. and Zanatta, N. (2000). New benzodiazepines 
alter acetylcholinesterase and ATPDase activities. Neurochemistry Research, 25: 949-955.

Shinomol, G. K. (2011). Bacopa monnieri modulates endogenous cytoplasmic and mitochondrial oxidative markers in prepubertal mice brain. Phytomedicine, 18 (4): 317-326.

Xu, Y., Colletier, J. P., Jiang, H., Silman, I., Sussman, J. L. and Weik, M. (2008). Induced-fit or preexisting equilibrium dynamics? Lessons from protein crystallography and MD simulation on acetylcholinesterase and implications for structure-based drug design. Protein Science, 17:601-605

Yang, H. J., Kwon, Y. D., Kim, J. M., Kang, S., Moon, R. N., Daily, W. J. and Park, S.
(2015). Red peppers with moderate and severe pungency prevent the memory deficit and hepatic insulin resistance in diabetic rats with Alzheimer's disease. Nutrition Metabolism(London), 12:1-12.

Yasser, A. K., Iman, M. M., Haitham, S. M., Neveen, A. N. and Heba, S. A. E. (2016). A study on the possible therapeutic role of Panax ginseng extract against a rat model of Parkinson's disease induced by intrastriatal rotenone injection. International Journal of Clinical and Experimental Medicine, $9(2): 3831-3841$.

Zimmermann, H. (2000). Extracellular metabolism of ATP and other nucleotides. Naunyn Schmiedeberg's Archives of Pharmacology, 362:299e309. 